\title{
Proteine im Wein - Nahrungsmittelallergie durch Wein?
}

\author{
Wine Proteins - Food Allergy Through Wine?
}

Autoren

Institut
P. Wigand, H. Decker

Institut für Molekulare Biophysik, Johannes Gutenberg-Universität Mainz

\section{Bibliografie}

DOI http://dx.doi.org/ 10.1055/s-0030-1256015

Online-Publikation: 9. 12. 2010

Akt Dermatol 2011; 37:

42-46 ๑ Georg Thieme

Verlag KG Stuttgart · New York ISSN 0340-2541

\section{Korrespondenzadresse} Dr. rer. nat. Petra Wigand Institut für Molekulare Biophysik Johannes Gutenberg-Universität Mainz

Jakob Welder-Weg 26

55128 Mainz

wigandp@uni-mainz.de

\section{Zusammenfassung \\ $\nabla$}

Wein enthält neben vielen weiteren Inhaltsstoffen Proteine in geringen Mengen, welche im Prinzip allergen wirken können. Die Proteine gelangen vor allem über die Traube in den Wein, können jedoch auch von Hefen und Bakterien freigesetzt werden. Der Hauptanteil der Weinproteine sind der Klasse der PR (Pathogenese-bezogenen)Proteine zuzuordnen. Einige dieser Proteine werden als potenzielle Allergene diskutiert. Das ein-

\section{Einleitung}

In den vergangenen Jahren wurden immer mehr Nahrungsmittelallergien nachgewiesen, wobei als Allergene oft Proteine identifiziert wurden [1]. Ein seit Jahrtausenden bekanntes Genuss- und Nahrungsmittel ist Wein. Tatsächlich gibt es einige Fallstudien über das Auftreten einer Weinallergie, die in der Regel mit schwerwiegenden Symptomen einhergeht [2-16], aber eine umfassende epidemiologische Untersuchung gibt es nicht.

Dabei werden auch Proteine als mögliche Allergene angenommen, die in geringen Mengen im Wein vorkommen. Die Proteine gelangen vor allem über die Traube in den Wein, können jedoch auch von Hefen und Bakterien in den Wein freigesetzt werden [17-19].

Durch den Einsatz der Massenspektrometrie zur Identifizierung von Proteinen wurden in den vergangenen fünf Jahren Erkenntnisse zur Proteinzusammensetzung im Wein gewonnen [18-22]. Der Hauptanteil der Weinproteine sind der Klasse der PR (Pathogenese-bezogenen)-Proteine zuzuordnen, die in der Pflanze eine Abwehrfunktion ausüben und von der Weinbeere in den Wein übergehen [16-21].

Eine erste Untersuchung zum allergenen Potenzial der Weinproteine wurde von Pastorello et al. (2003) veröffentlicht. Sie konnten in der Wein- zige offiziell international registrierte Allergen der Weinbeere ist ein Lipid-Transfer-Protein (LTP). Dieses konnte auch in Portugieser und Dornfelder Rotwein nachgewiesen werden. Um die mögliche allergene Wirkung zu testen, wurde das LTP aus dem Wein aufgereinigt und zunächst biochemisch und biophysikalisch charakterisiert. Strukturelle Analysen zeigen eine Ähnlichkeit der drei potenziellen Weinallergene (LTP, Thaumatin-ähnliche Proteine, Endochitinase) mit bekannten Nahrungsmittelallergenen.

beere drei Proteine identifizieren, die mögliche Allergene darstellen: das Lipid-Transfer-Protein (LTP), ein Thaumatin-ähnliches Protein (TLP) sowie eine Klasse 4-Endochitinase. In der gleichen Studie zeigten jedoch Weinallergiker allergische Reaktionen nur gegenüber der Endochitinase nach dem Konsum von jungem Rotwein [9]. Vassilopoulou et al. (2007) hingegen konnten das LipidTransfer-Protein als Auslöser einer Allergie gegenüber Weinbeeren bestätigen, während Thaumatin-ähnliche Proteine und Endochitinasen kein allergenes Potenzial besitzen [23]. Nach wie vor ist unklar, in welchem Umfang welche Proteine aus dem Wein zum Auslösen einer klinischen Reaktion führen können.

Das Lipid-Transfer-Protein ist ein bekanntes Nahrungsmittelallergen. Insbesondere in den mediterranen Ländern ist es als Auslöser für allergische Reaktionen auf Früchte der Rosaceen-Familie bekannt [23-25]. So stellt das Lipid-TransferProtein das einzige in der offiziellen Datenbank geführte Allergen der Weinbeere (Vitis Vinifera) dar [27].

Ziel unserer Untersuchungen ist die Aufreinigung sowie die biochemische, biophysikalische und strukturelle Charakterisierung von Weinproteinen. Damit bietet sich die Möglichkeit, mit den gereinigten Weinproteinen die allergene Wirkung dieser Proteine zu testen. 


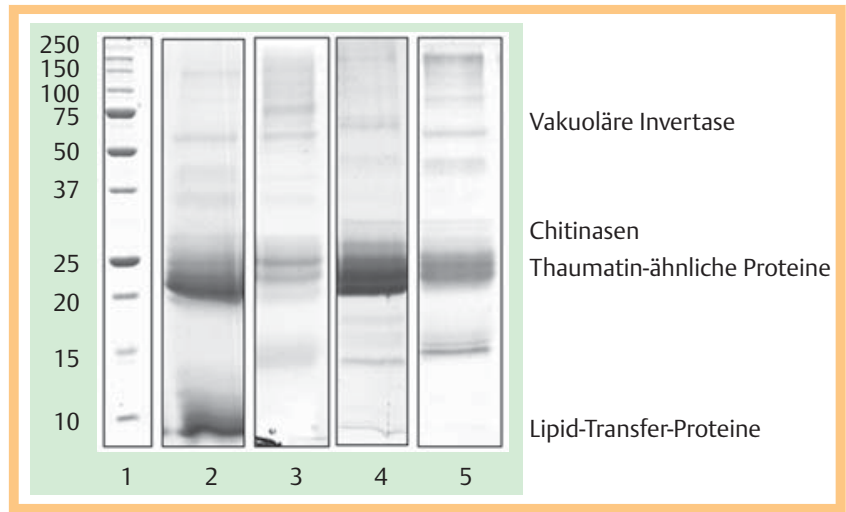

Abb. 1 Coomassie-gefärbte SDS-PAGE [12,5\%] deutscher Weine. 1 Molekulargewichtsmarker, 2 Portugieser Rotwein, 3 Riesling, 4 Sauvignon Blanc, 5 Gewürztraminer.

\section{Material und Methoden}

Biochemische und strukturelle Charakterisierung der Weinproteine

Weine: Die Weine wurden vom Weingut Fleischer (Mainz) bezogen oder im Supermarkt erworben. Als Weißweine wurden Riesling 2006, Sauvignon Blanc 2006 und Gewürztraminer 2006 (alle aus Rheinhessen) eingesetzt und als Rotweine Portugieser 2005 aus der Pfalz sowie Dornfelder 2007 aus Rheinhessen.

Konzentrierung der Proteine: Für die Dialysen werden Spectra/ Por $^{\circledR}$-Dialyseschläuche aus regenerierter Cellulose mit einer Ausschlussgrenze von 3,5 kDa verwendet. Die Dialysen erfolgen bei $4^{\circ} \mathrm{C}$ unter ständigem Rühren für mindestens 72 Stunden gegen destilliertes Wasser bei mindestens zweifachem Wechsel des Wassers pro Tag. Im Anschluss werden die Weinproben im Rundkolben mittels Spin-Freezing in einem Kältebad bei $-30^{\circ} \mathrm{C}$ gefroren, das Trocknen findet bei einem Druck von 0,940 mbar über einen Zeitraum von 24 Stunden statt.

Fällung mit Polyvinylpyrrolidon: Zur Abtrennung der Polyphenole werden die lyophilisierten Weinproben mit PVP (Polyclar ${ }^{\circledR}$ AT) gefällt. Hierfür werden $300 \mathrm{mg}$ der Weinprobe in $30 \mathrm{ml}$ Puffer gelöst und mit $3 \mathrm{~g}$ PVP versetzt.

Auftrennung mittels SDS-PAGE: Die diskontinuierliche SDS-PAGE wird nach der Methode von Laemmli durchgeführt [28]. Eingesetzt wird ein 3\%iges Sammelgel und ein 12,5 oder 15\%iges Trenngel. Die Proteinproben werden im Verhältnis 1:1 mit Denaturierungspuffer (TRIS-Puffer mit 20\% [v/v] Glycerin, 10\% [v/v] $\beta$-Mercaptoethanol, 4\% [w/v] SDS, Spatelspitze Bromphenolblau) gemischt und nach einer zehnminütigen Denaturierung bei $95^{\circ} \mathrm{C}$ auf das Gel aufgetragen. Die Elektrophorese wird bei $120 \mathrm{~V}$ bei Raumtemperatur durchgeführt.

Aufreinigung mittels Kationenaustauschchromatografie: Die Kationenaustauschchromatografie wird mit einer UNO-S6-Säule (Bio-Rad) durchgeführt. Als Puffer wird $50 \mathrm{mM}$ Zitratpuffer mit einem pH-Wert von 3,0 eingesetzt, eluiert wird mit 1-molarem NaCl-Gradient.

Identifizierung: Die Identifizierung erfolgt mittels LC-MS/MS am Institut für Immunologie der Universitätsmedizin Mainz in der AG Schild durch Dr. Tenzer. Hierzu werden die Coomassie-gefärbten Proteinbanden aus der SDS-PAGE ausgeschnitten und mit Trypsin verdaut [18].

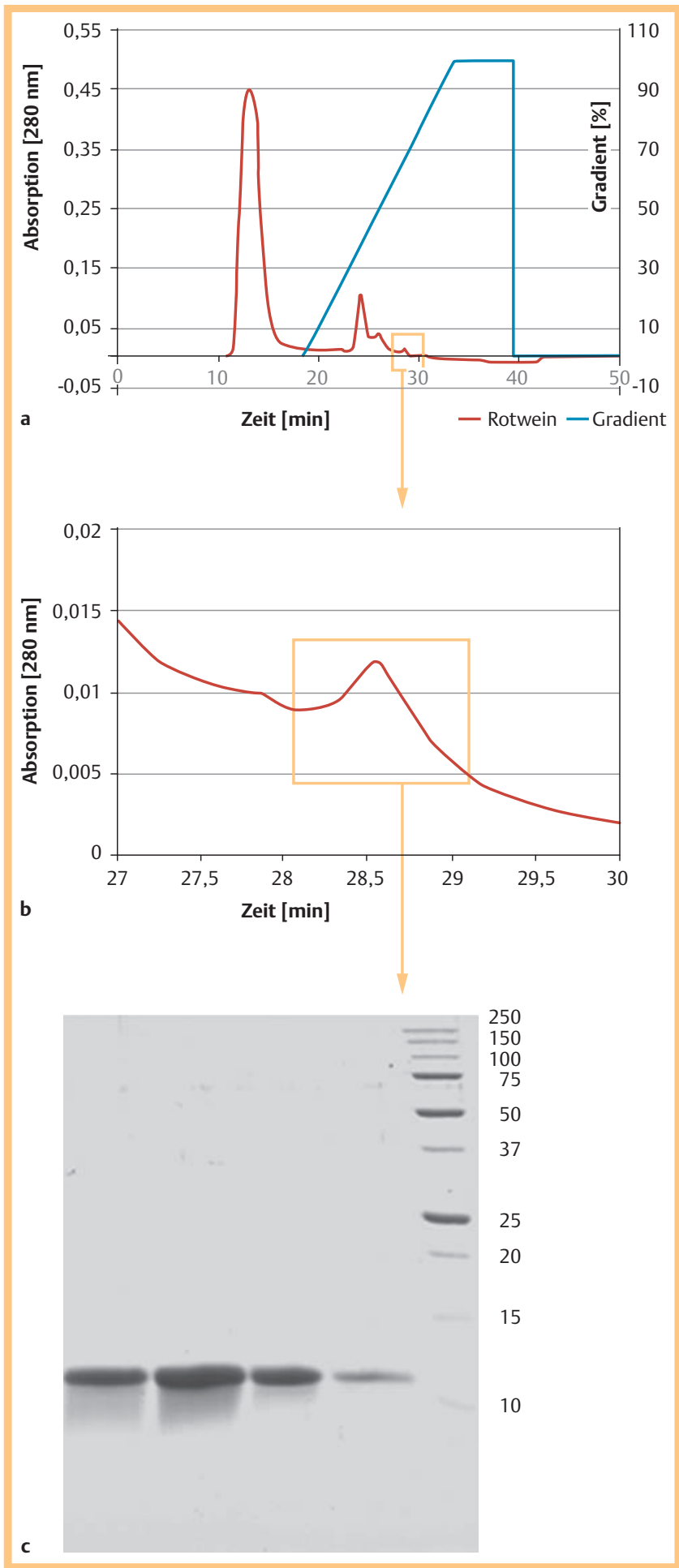

Abb. 2 Aufreinigung des LTP aus Dornfelder mittels Kationenaustauschchromatografie. Eingesetzt wurde dialysierter und gefriergetrockneter Dornfelder 2007 nach PVP-Fällung. a Chromatogramm des Kationenaustauschs, durchgeführt bei einem pH-Wert von 3,0. 1: Thaumatin-ähnliche Proteine; 2: Lipid-Transfer-Protein; b Ausschnitt aus a mit der Elution des Lipid-Transfer-Proteins; c Coomassie-gefärbte SDS-PAGE [15\%] mit den Fraktionen aus $\mathbf{b}$.

\section{Ergebnisse}

Biochemische und strukturelle Charakterisierung Bei allen untersuchten Weinsorten (deutsche Rot- und Weißweine) ist der Hauptanteil der Proteine den PR-Proteinen (pathoge- 


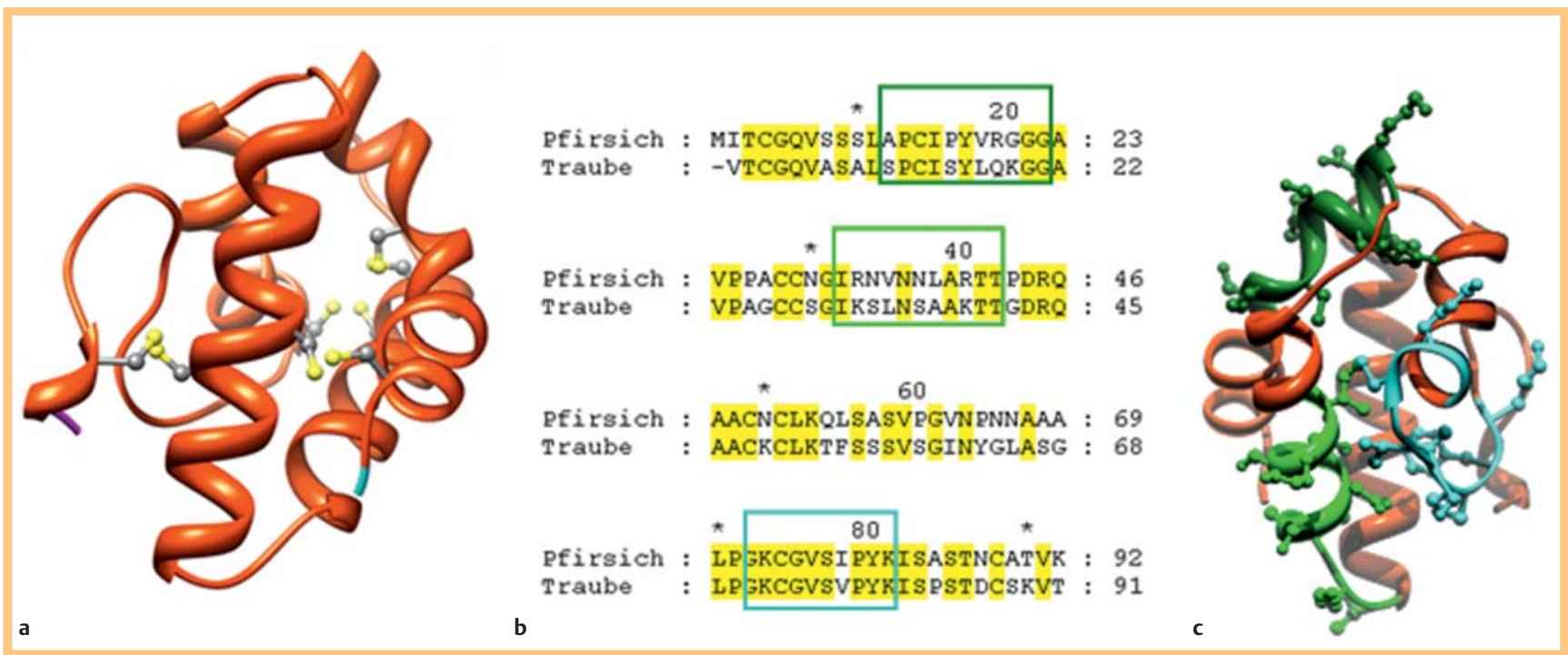

Abb. 3 Proteinmodell des LTP (Isoform 4) aus der Weinbeere. Erstellt mit Swiss Model und bearbeitet mit Chimera. a Das Homologie-Modell des LTP. Die stabilisierenden Disulfidbrücken sind in gelb eingezeichnet. b Sequenzalignment des LTP aus der Weinbeere mit dem LTP aus Pfirsich, erstellt mit ClustalX. Der hohe Grad an Sequenzidentität ist im Vergleich der beiden Sequenzen zu erkennen; c Die potenziellen Epitope des pru p 3 übertragen auf die Struktur des LTP aus der Weinbeere. Aus Gründen der Übersichtlichkeit wurde die Struktur um $90^{\circ}$ gegenüber a gedreht.

nensis-related proteins) zuzuordnen, die sich im Molekulargewichtsbereich von 10 bis $27 \mathrm{kDa}$ befinden. Dies zeigt die Gelelektrophorese am Beispiel von Portugieser Rotwein, Riesling, Sauvignon und Gewürztraminer $(\bullet$ Abb. 1$)$.

In einem Portugieser Rotwein konnte ein Lipid-Transfer-Protein $\left(\mathrm{M}_{\mathrm{r}} 11 \mathrm{kDa}\right)$, Thaumatin-ähnliche Proteine $\left(\mathrm{M}_{\mathrm{r}} 20-24 \mathrm{kDa}\right.$ sowie die Klasse-4-Endochitinase $\left(\mathrm{M}_{\mathrm{r}} 27 \mathrm{kDa}\right)$ identifiziert werden [18]. Da das Lipid-Transfer-Protein als einziges Protein der Weinbeere als Allergen in der offiziellen Allergendatenbank geführt wird, beschränkten wir unsere folgenden Untersuchungen deshalb auf dieses Protein. Es ist vor allem im Rotwein enthalten, da es in der Schale der Weinbeere vorkommt und somit bei der Maischegärung in den Wein übergehen kann, während es bei der Mostgärung des Weißweins vorher weitgehend mit dem Trester abgetrennt wird.

Diese Überlegung zeigt sich auch bei der gelelektrophoretischen Auftrennung der Weinproteine. Das Lipid-Transfer-Protein mit einer molekularen Masse von etwa $11 \mathrm{kDa}$ ist im Rotwein gut als Bande zu erkennen, während es in den Weißweinen nicht zu erkennen ist ( Abb. 1).

Auch in Dornfelder Rotweinen verschiedener Jahrgänge konnte das LTP nachgewiesen werden. Deshalb setzten wir für die weitere Charakterisierung exemplarisch Dornfelder Rotwein des Jahrgangs 2007 aus Rheinhessen ein.

\section{Aufreinigung des Lipid-Transfer-Proteins aus Rotwein $\nabla$}

Da sich das Lipid-Transfer-Protein im Vergleich zu den anderen Weinproteinen durch einen hohen (theoretischen) isoelektrischen Punkt von etwa 9 auszeichnet, wird zur Aufreinigung die Kationenaustauschchromatografie eingesetzt. Das Chromatogramm zeigt, dass aus einem Dornfelder 2007 nach Gefriertrocknung und PVP-Fällung verschiedene Proteine aufgereinigt werden. Während zunächst die Thaumatin-ähnlichen Proteine eluieren, wird als letztes Protein in einem kleinen Peak das LTP aufgereinigt ( Abb. 2). Dies ist in der SDS-PAGE dokumentiert und konnte durch eine massenspektrometrische Analyse bestätigt werden.

\section{Sequenz- und Strukturvergleich mit bekannten Allergenen $\nabla$}

Ein 3D-Modell des LTPs konnte mit bioinformatischen Methoden erstellt werden. Als Grundlage diente das LTP aus Pfirsich (pru p 3), für welches eine Struktur beschrieben ist. Dieses LTP und das aus der Weinbeere (vit v 1, entspricht der Isoform 4) zeigen eine hohe Sequenzähnlichkeit [28]. Somit konnte auf der Grundlage dieser Struktur mit Kenntnis der Sequenz des LTP (Isoform 4) eine Struktur für das LTP aus dem Wein erstellt werden. Dies erfolgte durch Homologie-Modellierung mithilfe von Swiss Model [27-29]. Die Struktur ist in Abb. 3 a dargestellt.

Die Strukturen der verschiedenen Lipid-Transfer-Proteine sind sehr ähnlich. Insbesondere die acht Cysteine, welche vier Disulfidbrücken ausbilden, sind konserviert. Die hohe Sequenzähnlichkeit von $47 \%$ (Identität) und 62\% (physiko-chemische Ähnlichkeit) lässt nicht nur auf eine sehr ähnliche Struktur ( $\mathbf{A} \mathbf{b b}$. 3b und 3c) schließen. Tatsächlich gibt es drei Bereiche auf der Proteinoberfläche des LTP, die als allergene Determinanten diskutiert werden [32]. In einem dieser Bereiche gibt es nur einen Aminosäureaustausch durch einen isofunktionellen Seitenrest von Valin zu Isoleucin.

\section{Diskussion \\ $\nabla$}

Unsere Untersuchungen zeigen, dass in den von uns untersuchten deutschen Weinen tatsächlich potenzielle Allergene enthalten sind. Die drei nachgewiesenen PR-Proteine werden deshalb als potenziell allergene Proteine betrachtet, da sie drei Proteingruppen zugeordnet werden können, die als Nahrungsmittelallergene bekannt sind ( $\bullet$ Abb. 4). 


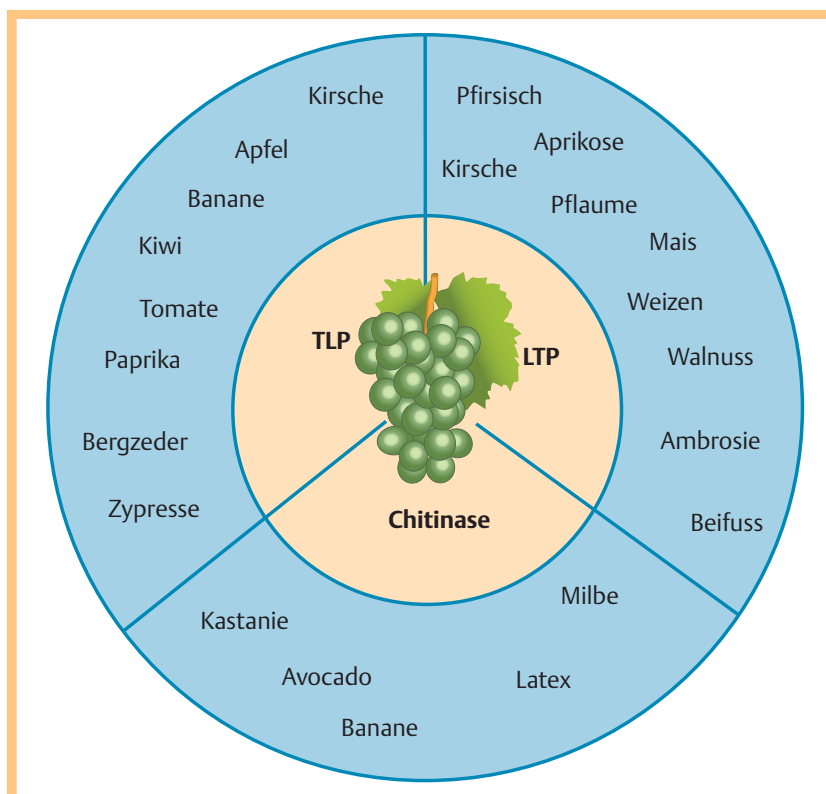

Abb. 4 Schematische Zuordnung der drei nachgewiesenen PR-Proteine zu bekannten Nahrungsmittelallergenen.

Das Lipid-Transfer-Protein konnte bereits in einem Chardonnay Wein aus Japan identifiziert werden [18]. Unsere Untersuchungen weisen das Vorkommen des LTP auch in deutschen Rotweinen nach, wobei entsprechende Untersuchungen aus anderen Weinregionen noch anstehen. Lipid-Transfer-Proteine sind als Nahrungsmittelallergene vieler Pflanzen, auch unter dem Begriff des LTP-Syndroms, bekannt und klinische Reaktionen treten vor allem in den mediterranen Ländern auf [21 -24]. Das LTP vit v 1 der Weinbeere zeigt hohe Sequenzidentität zu dem Hauptallergen pru p 3 aus Pfirsich. Zwischen diesen Proteinen wurden bereits Kreuzreaktionen beschrieben [8]. Diese Ähnlichkeit lässt auch auf ein ähnliches allergenes Verhalten schließen. Inwieweit das LTP aus dem Wein als Allergen wirkt, muss allerdings durch klinische Versuche erst bestätigt werden. Zudem bedarf es noch der Klärung, ob das LTP der Weinbeere auch eine primäre Sensibilisierung hervorrufen kann, d.h. auch unabhängig von einer Pfirsichallergie auftritt.

Für die klinischen Untersuchungen müssen die Proteine erst aufgereinigt werden. Für das Lipid-Transfer-Protein konnten wir durch eine PVP-Fällung mit anschließender Kationenaustauschchromatografie eine Isolierung mit hoher Reinheit erreichen. Unserem Wissen nach ist dies die erste Arbeit, die eine Isolierung des LTP aus Wein beschreibt. Unsere aktuellen Untersuchungen konzentrieren sich auf die biophysikalische Charakterisierung des LTPs, um auch zu klären, ob durch die Weinbereitung strukturelle Änderungen hervorgerufen werden und damit die Induktion einer Allergie ausbleibt und wenn, weshalb.

Wir möchten hier besonders betonen, dass nicht ausschließlich rheinhessische bzw. deutsche Weine Proteine mit potenziell allergenem Charakter enthalten, sondern alle von uns untersuchten Weine [16]. Dies wird auch durch die Literatur bestätigt [9,19-23]. Sollten unsere Untersuchungen ergeben, dass die Weinproteine allergische Reaktionen auslösen können, werden unsere Ergebnisse dazu beitragen, den Winzern Hinweise zu geben, das an sich sichere Produkt Wein auch für Allergiker noch sicherer zu machen.

\section{Danksagung}

$\nabla$

Wir danken dem Weingut Fleischer aus Mainz für die freundliche Überlassung der Weine und der AG Schild/Tenzer für die Durchführung der massenspektrometrischen Analysen.

Die Untersuchungen werden von der Stiftung Rheinland-Pfalz für Innovation und der Johannes Gutenberg-Universität Mainz gefördert.

\section{Abstract}

\section{Wine Proteins - Food Allergy Through Wine?}

Proteins are minor constituents in wine. Most of the wine proteins derive from the grape, some are released from yeast or bacteria. The pathogenesis-related proteins represent the main proteins in all of the wines. Some of them are discussed as potential grape and wine allergens with the lipid transfer protein being the only officially accepted allergen in grapes. This protein could be identified in Portugieser and Dornfelder red wine, whereas its presence in white wine is rare. To investigate the allergenic potential, the lipid transfer protein was purified and biochemically and biophysically characterised. Structural analyses show similarity between the three potential wine allergens with known food allergens.

\section{Literatur}

1 Schäfer T. Epidemiologie der Nahrungsmittelallergie in Europa. Ernährung 1980; 2: 4-9

2 Clayton DE, Busse W. Anaphylaxis to wine. Clin Allergy 1980; 10: 341 343

3 Giannoccaro F, Munno G, Riva G et al. Oral allergy syndrome to grapes. Allergy 1998; 53: $451-452$

4 Vaswani SK, Chang BW, Carey RN, Hamilton RG. Adult onset grape hypersensitivity causing life threatening anaphylaxis. Ann Allergy Asthma Immunol 1999; 83: 25 - 26

5 Bircher A, Bigliardi JP, Yilmaz B. Anaphylaxis resulting from selective sensitization to Americana grapes. J Allergy Clin Immunol 1999; 104: $1111-1113$

6 Karakaya G, Kalyoncu AF. Allergy to grapes. Ann Allergy Asthma Immunol 2000; 84: 265

7 Rodriguez A, Trujillo MJ, Matheu V et al. Allergy to grape: a case report. Pediatr Allergy Immunol 2001; 12: 289-290

8 Senna G, Mistrello G, Roncarolo D et al. Exercise-induced anaphylaxis to grape. Allergy 2001; 56: 1235-1236

9 Pastorello EA, Farioli L, Pravettoni V et al. Identification of grape and wine allergens as an endochitinase 4 , a lipid-transfer protein, and a thaumatin. J Allergy Clin Immunol 2003; 111: 350-359

10 Rodriguez A, Matheu V, Trujillo MJ et al. Grape allergy in paediatric population. Allergy 2004; 59: 364

11 Borghesan F, Basso D, Chieco Bianchi $F$ et al. Allergy to wine. Allergy 2004; 59: 1135 - 1136

12 Kalogeromitros DC, Rigopoulos D, Gregoriou SG et al. Asymptomatic sensitisation to grapes in a sample of workers in the wine industry. Occup Environ Med 2004; 61: 709-711

13 Kalogeromitros DC, Makris MP, Gregoriou SG et al. Grape anaphylaxis: a study of 11 adult onset cases. Allergy and Asthma Proceedings 2005; 26: $53-58$

14 Kalogeromitros DC, Makris MP, Gregoriou SG et al. Sensitization to other foods in subjects with reported allergy to grapes. Allergy Asthma Proc 2006; 27: $68-71$

15 Sbornik M, Rakoski J, Mempel $M$ et al. IgE-mediated type-I-allergy against red wine and grapes. Allergy 2007; 62: 1339-1340

16 Schäd SG, Trcka J, Vieths S et al. Wine anaphylaxis in a German patient: IgE-mediated allergy against a lipid transfer protein of grapes. Int Arch Allergy Immunol 2005; 136: 159-164 
17 Wigand P, Decker H. Proteine im Wein. Deutsche Lebensmittel-Rundschau 2007; 2 : $52-57$

18 Wigand P, Tenzer S, Schild H, Decker H. Protein composition of Red Wine in comparison with Rosé Wine and White Wine by Electrophoresis and HPLC/Mass Spectrometry. J Agric Food Chem 2009; 57: 43284333

19 Kwon SW. Profiling of soluble proteins in wine by nano-high-performance liquid chromatography/tandem mass spectrometry. J Agric Food Chem 2004; 52: 7258 - 7263

20 Cilindre C, Jegou S, Hovasse A et al. Proteomic approach to identify champagne wine proteins as modified by Botrytis cinerea infection. J Proteome Res 2008; 7: 1199-1208

21 Okuda T, Fukui M, Takayanagi T, Yokotsuka K. Characterization of major stable proteins in Chardonnay wine. Food Science Technol Res 2006; 12: $131-136$

22 Flamini R, De Rosso M. Mass spectrometry in the analysis of grape and wine proteins. Expert Rev Proteomics 2006; 3: 321 - 331

23 Vassilopoulou E, Zuidmeer L, Akkerdaas J et al. Severe immediate allergic reactions to grapes: part of a lipid transfer protein-associated clinical syndrome. Int Arch Allergy Immunol 2007; 143: 92 - 102

24 Salcedo G, Sanchez-Monge R, Diaz-Perales A et al. Plant non-specific lipid transfer proteins as food and pollen allergens. Clin Exp Allergy 2004; 34: 1336 - 1341
25 Pastorello EA, Robino AM. Clinical role of lipid transfer proteins in food allergy. Mol Nutr Food Res 2004; 48: 356 - 362

26 Salcedo G, Sanchez-Monge R, Barber D, Diaz-Perales A. Plant non-specific lipid transfer proteins: an interface between plant defence and human allergy. Biochim Biophys Acta 2007; 1771: 781 - 791

27 IUIS Allergen Nomenclature Sub-Committee ALLERGEN NOMENCLATURE. www.allergen.org; Stand: 18.10 .2010

28 Laemmli UK. Cleavage of structural proteins during the assembly of the head of bacteriophage T4. Nature 1970; 227: 680-685

29 Pasquato N, Berni R, Folli C et al. Crystal structure of peach Pru p 3, the prototypic member of the family of plant non-specific lipid transfer protein pan-allergens. J Mol Biol 2006; 356: 684-694

30 Arnold K, Bordoli L, Kopp J, Schwede T. The SWISS-MODEL Workspace: A web-based environment for protein structure homology modelling. Bioinformatics 2006; 22: 195-201

31 Kiefer F, Arnold K, Künzli $M$ et al. The SWISS-MODEL Repository and associated resources. Nucleic Acids Research 2009; 37: D387-D392

32 Peitsch MC. Protein modeling by E-mail. Nature Biotechnology 1995; 13: $658-660$

33 Garcia-Casado G, Pacios LF et al. Identification of IgE-binding epitopes of the major peach allergen Pru p 3. J Allergy Clin Immunol 2003; 112: $599-605$ 